\title{
ANALYTICAL MODELING OF BATTERY CYCLE LIFE
}

\author{
S. Gross
}

Boeing

What I have attempted to do is look at the problem of battery cycle life from the standpoint of developing a simple analytical model that would be related to the physical and chemical processes that are involved in batter wear and failure.

The major assumption in this attempt was that in the cycling regime, cells will gradually lose capacity until the remaining capacity is depleted. And at that point the discharge cannot be supported.

For those cells which fail more abruptly, it is assumed that the processes which are severe in the degrading capacity are equally severe in the abrupt failures.

Furthermore, let's assume that the battery wearout consists of time-dependent chemical degradation and physical damage that is caused by cycling.

The model consists of a cell of unit capacity that is being discharged to a depth of discharge $\mathrm{D}$ when it is new, with a remaining capacity of 1 minus $\mathrm{D}$. And the capacity loss that occurs during cycling is then 1 minus $\mathrm{D}$, and that is equal to the chemical degradation loss which is time dependent, A times the number of cycles. $\mathrm{N}$ can be the number of cycles for cycle-based analysis, or $\mathrm{T}$ for time-based analysis for unit cycling time; plus the cycling degradation loss which is constant times the amount of discharge, the DOD times the number of cycles-to-failure which is the turnaround of the cell capacity.

Furthermore, it can be assumed that this coefficient B is dependent upon the DOD. It can be assumed to be some function of the DOD. I have assumed it is a possibility of being an exponential function and therefore entering into the coefficient on D, coefficient of $M$. For the case where it is not dependent on DOD, M would be simply one. And in that case, this term of one less $D$ divided by $\mathrm{D}$ without the coefficient $\mathrm{C}$, and $\mathrm{B}$ would be of course the group that Dr. Lander has been using.

(Figure 5-56)

To test this out, I have used the data that is readily available from Crane tests. This is the old data, not the accelerated life data, but the data many people have analyzed and reduced.

This data is shown here, this plotting cycle life against the DOD based on the rated capacity which is the top lines for each of the three temperatures. But for this analysis, we need the real capacity rather than rated capacity, and that has been worked to produce the lines just below, giving the real capacity. This is based on an assumption or based on an experience of a typical relationship between real initial capacity and rated capacity. 


\section{(Figure 5-57)}

To check out this model, I have taken the Crane data. This is, of course, the 1.5-hour orbit data. The zero-degree data that was shown in the previous chart is given in red. This chart plots this function which has been fitted to match this data, the Crane data for variable exponents: $\mathrm{N}$ equal to 1 or 2 .

And it would seem it doesn't make very much difference what $N$ is, except in the areas of low depth of discharge and fairly deep depth of discharge rated at 90-percent range.

(Figure 5-58)

Now, assuming that $M$ is equal to 2, we made the correlation for all three temperatures, and produced this type of chart. The thing we see is that the straight line, log DOD relationship that is often extrapolated on the Crane data or other test data, does not appear to hold in this kind of correlation.

We have a falling over on both the low DOD and the deep DOD side. For the case of N equals 1 , it would be less of a fallover. In the case of A equals zero, this of course goes up to infinity.

(Figure 5-59)

The next question then comes up, what is the effect of temperature?

Taking the first chart on Crane cycle-life data and cross plotting it, we get cycle life as a function of temperature plotted linearly for two depths of discharge, 0.2 and $0.5,20$ percent and 50 percent.

Unfortunately, we have only three data points, only three temperatures, so we can't get a very good curve, but the two things that are apparent when you look at it more carefully is that the slope from zero to 25 differs as a function of the DOD. This slope does not equal that slope. And secondly, there is a change in slope at the higher temperatures, 25 to 40 degrees.

(Figure 5-60)

Now, when we plot the coefficients, A and B on an Arrhenius type plot, coefficients against reciprocal temperatures, again we see the same discontinuity in slope. This is the curve for $\mathbf{A}$. $A$ is the coefficient in the equation.

(Figure 5-61)

We have the same thing for $\mathrm{B}$, also a discontinuity in the slope at the higher temperatures.

These last three charts all show that at the high temperature the degradation processes are occurring at a rate which is faster than would be determined by an Arrhenius type dependence, and certainly, it is not linear in temperature. 
This means there are different degradation processes occurring at the high temperature, or at least occurring at a significantly higher rate than we have with the low temperature.

So, as far as old Crane data is concerned, it would not be valid to do accelerating tests at high temperature without taking this into account.

\section{DISCUSSION}

RITTERMAN: You showed some very early failures at the very high DOD, almost close to zero cycles at 100-percent DOD, if I read that correctly?

GROSS: Yes. That was what the analytical model predicts.

RITTERMAN: That is the model. It wasn't in actuality? DOD.

GROSS: No, the actuality is fitted only to the limited DOD range, roughly 20- to 50-percent

RITTERMAN: How would you define failure?

GROSS: Failure in this case is defined the same way it was defined for the Crane tests where the data came from. For those tests it was defined as failure to provide 1.0 volt or failure to deliver a specified amount of capacity.

RITTERMAN: Was that 100-percent DOD, or you are bound to fail?

GROSS: The model predicts that at any time you are discharging, you are wearing something out. If you have 100-percent DOD and one cycle, but that one cycle did enough wearout so that in attempt to have cycle 2 , you cannot quite make 100 percent.

Now, in actual practice, there is a small amount of cycle improvement the first few cycles, and it would not be valid for those first few cycles because that's a second-order effect. That is not taken into account in the first few cycles.

RITTERMAN: The problem I am having is with the definition of failure. I think it would be more applicable to define it as a cell short or a severe failure in capacity.

GROSS: I have defined a cell failure as the inability to provide capacity required to maintain a prescribed DOD.

Secondly, I have further assumed for those failures which are premature, the early shorting failures (failures which happen fast and are included in some of the Crane data), that the same kind of stress that caused capacity degradation also caused the early failures. As you know, the Crane data is the average of a large number of points. 
MAURER: Sid, I think your last vugraph shows some of the dangers one can get into in extrapolating the data base beyond the range of the base. For example, in talks we heard this morning, the first three talks gave valid projections within that range.

But, if you would try to take those equations and extrapolate to geosynchronous conditions, for example, where we know that that equation would give a false indication of life, we would be predicting lifetimes of many tens of years. And we know that the lifetimes of those are shorter.

What you are doing is picking up the low activation energies first, and as you go to higher temperatures, you pick up higher activation energies. So that the lower part of your curve might be the mass transport mechanism that was discussed earlier this morning.

And the lesser part of your curve, where the higher temperatures are, would be into the higher activation energies as chemical reactions like nylon degradation which was taking place around 15 kilocalories in the tests that we have seen.

GROSS: I think there is no question about that. I think also there is no question that even though for some cycling conditions, time dependent processes are not large, they cannot be ignored and that an equation should be introduced. So, especially when you try to extrapolate them beyond a small amount of data, you at least have that parameter.

HAFEN: Sid, I would like to know if these failures were pack failures or individual cell failures. If they were pack failures, did you make an attempt to somehow correlate the individual ones?

GROSS: They were average cell failures of all of the cells. A pack failure would be the failure of the last cell in the pack. And this is the failure, average failure of the individual cells.

HAFEN: In other words, you take the cycle numbers and you divide them by the number of failures?

GROSS: What essentially is done is, the cycle life of an individual cell - of individual cells is plotted on a curve and you get a distribution, and you fit that distribution as well as you can, with, in this case only three lines; one line for each temperature.

VASANTH: Would it be possible to predict from your data any particular type of specific type of degradation process that will be taking place?

GROSS: If there had been more data points, more temperature data points than three, then it would have been possible to make some kind of estimates of the activation energy. But you just can't plot any, make any prediction on three points. You don't have enough data to draw curves that would give you the intersection that you need to get your activation action.

HALPERT: I did want to clarify a point that came up earlier in this morning's presentation by John Lander, who said manufacturers had a lot of flexibility. 
I did talk with John afterwards and with Ami Gupta, and the comment we tried to make is that the flexibility a manufacturer has is within the range of electroly te volume and concentration, that's all.

Secondly, in that regard, Ami Gupta had indicated the reason he was able to get some of the results he did get was because this particular lot of cells was very uniform.

As a matter of fact, a group of those flew in one of our spacecraft, the AE spacecraft, and it was also in three additional packs at Crane, the numbers of which I don't have at hand at the moment.

So he would not have been able to get this data without the uniformity of the packs. We don't want you to jump to any conclusions about flexibility in the manufacturing process. 


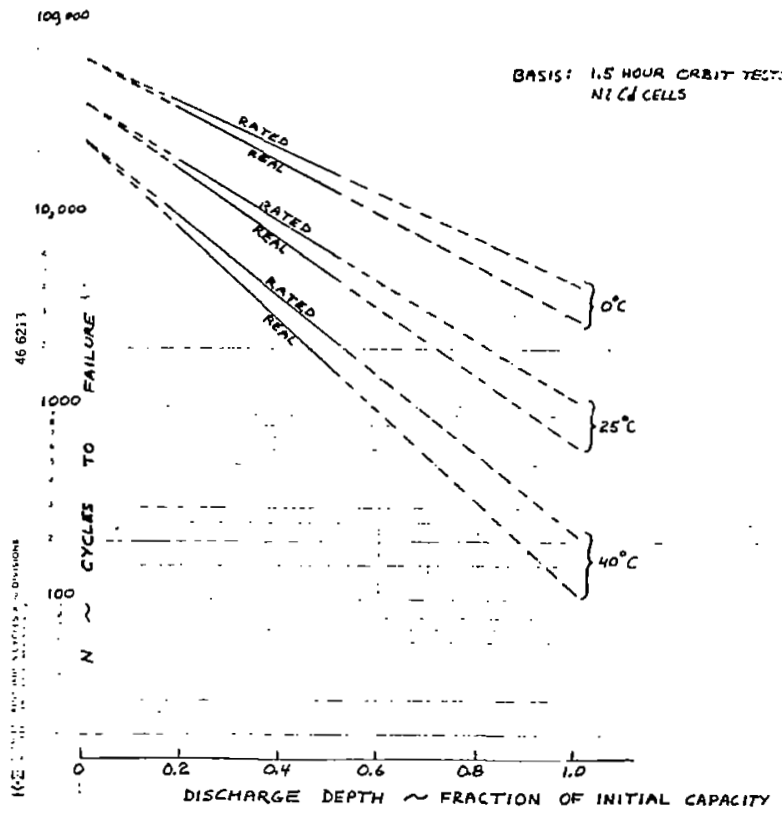

Figure 5-56

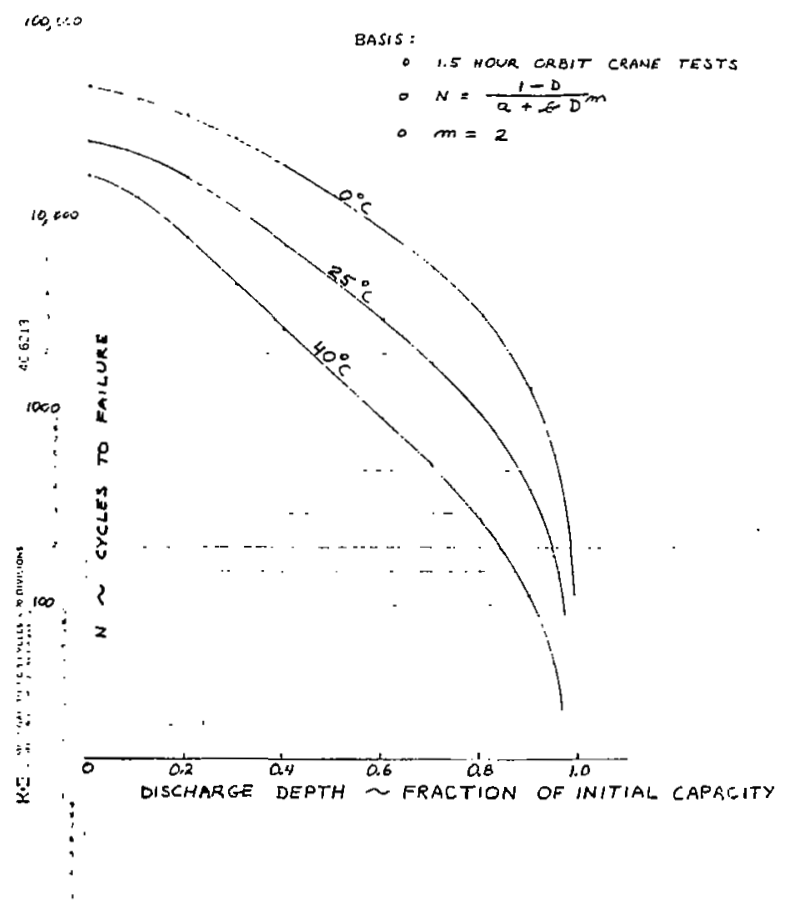

Figure 5-58

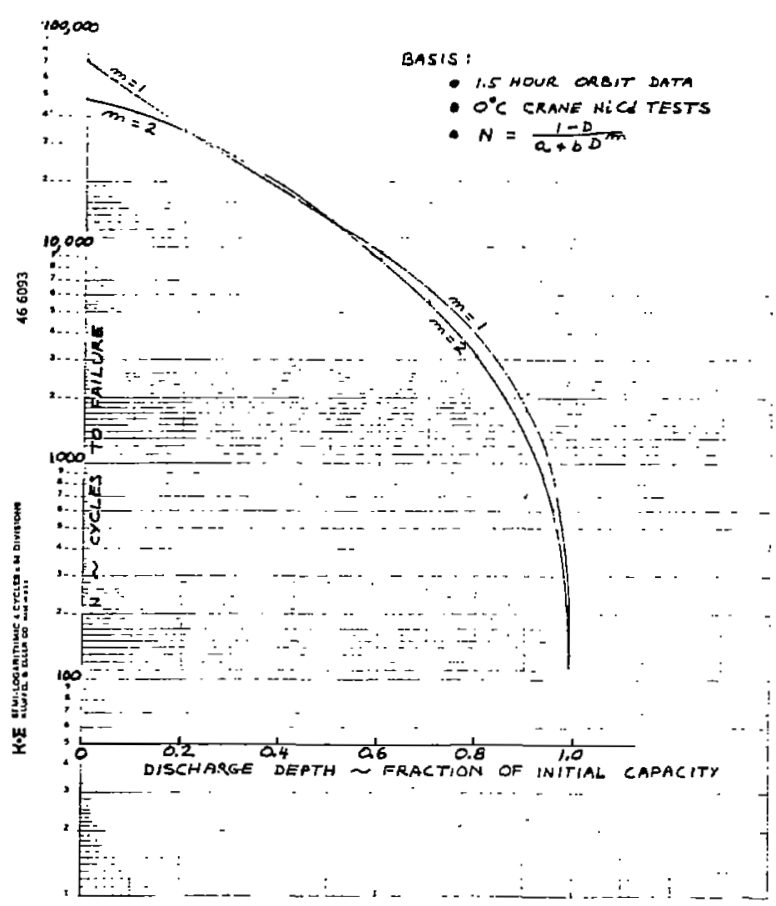

Figure 5-57

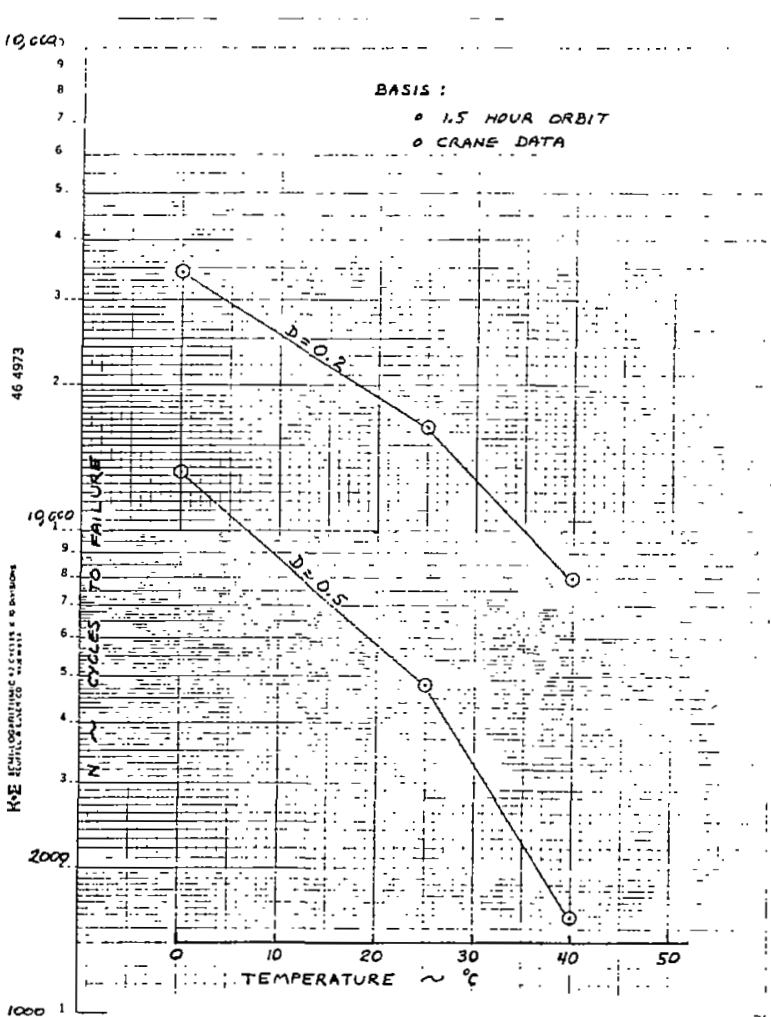

Figure 5-59 


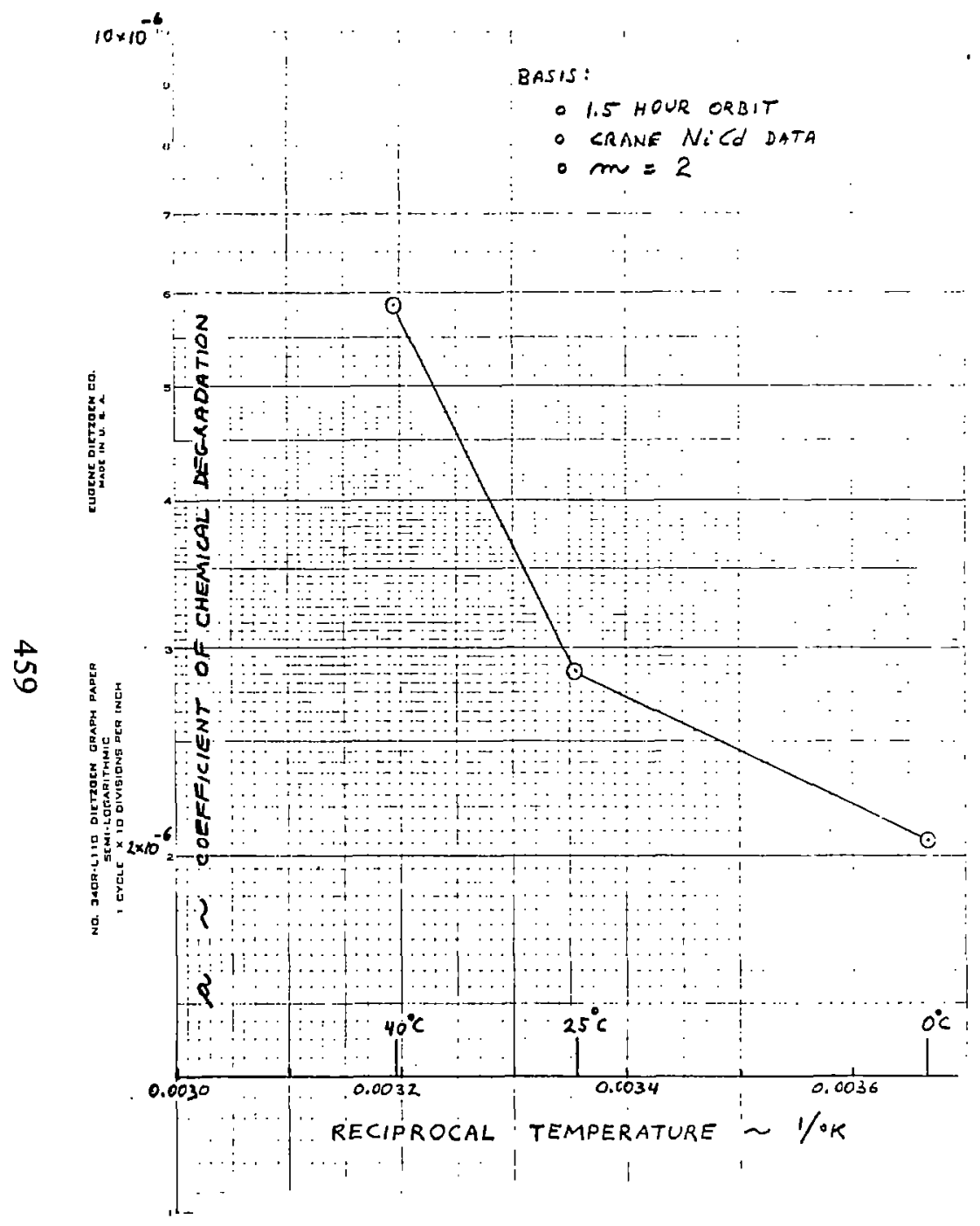

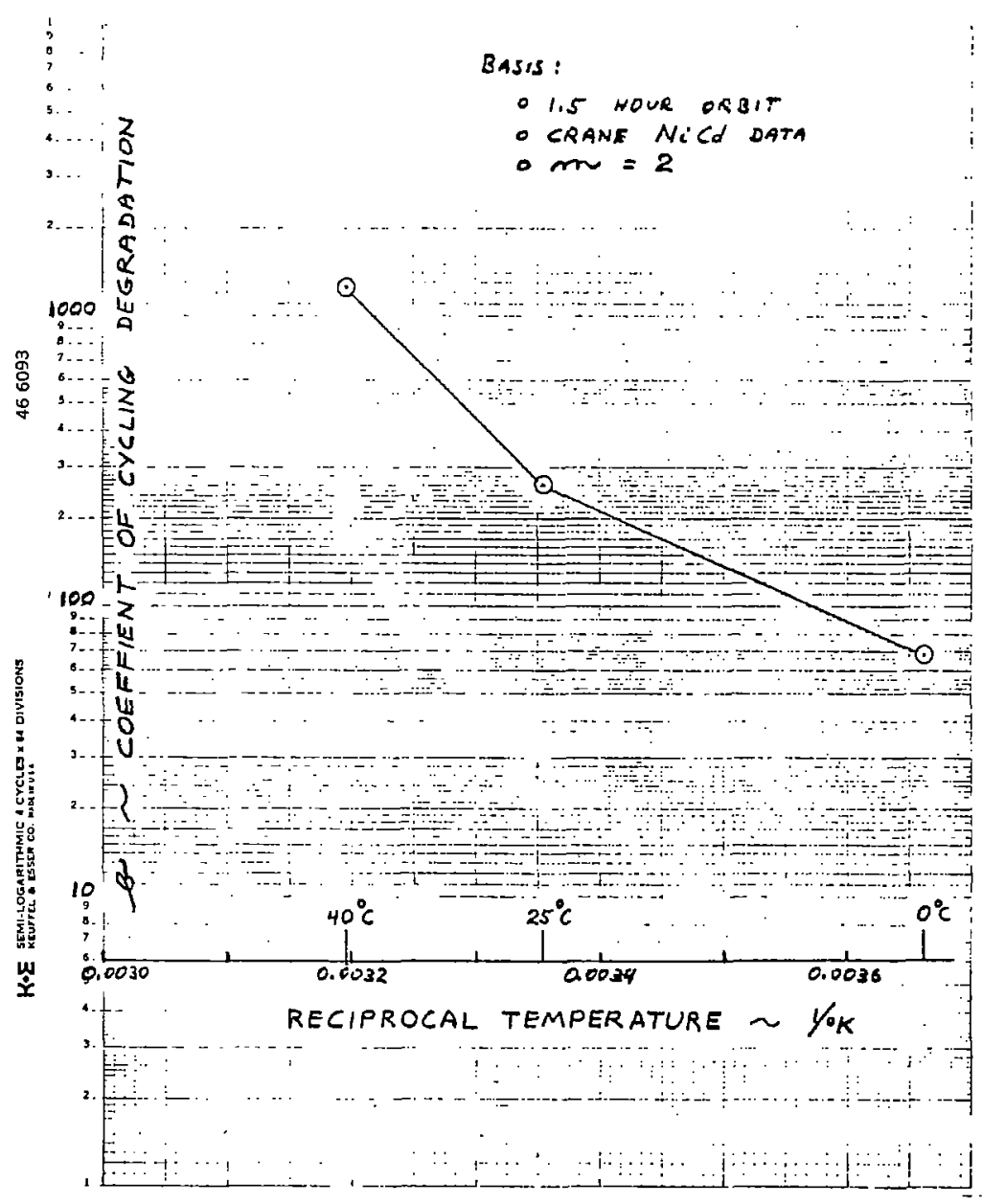

Figure 5-61

Figure 5-60 